\title{
HYPERCIRCLE ESTIMATES FOR NONLINEAR PROBLEMS
}

\author{
A. M. ARTHURS \\ (Received 31 October 1978) \\ (Revised 20 December 1978)
}

\begin{abstract}
Recent hypercircle estimates for non-linear equations are extended to include a new class of boundary value problems of monotone type. The results are illustrated by the boundary value problem for the equilibrium-free surface of a liquid with prescribed contact angle.
\end{abstract}

\section{Introduction}

The hypercircle, originally developed as a method for the approximate solution of certain linear boundary value problems [6], has recently been extended [4] to provide corresponding geometrical results for a class of non-linear boundary value problems. These problems involve equations of the type

$$
T^{*} T \phi=f(\phi),
$$

subject to certain boundary conditions, and many examples of such problems arise in mathematical physics.

Also of interest for applications, but not included in (1.1), are nonlinear problems of the form

$$
T^{*}[h(T \phi)]=g(\phi),
$$

where $h$ represents a prescribed non-linear function and $g$ may be linear or nonlinear. The problem of Plateau falls into this category, for instance, as do other equilibrium surface problems and problems of diffusion. Associated hypercircle estimates would therefore be useful for (1.2) and in this paper we present the results for those cases where they can be established, namely for various non-linear functions $h$ and linear functions $g$. Our results are illustrated by calculations for the non-linear partial differential equation that arises in the study of capillary surface problems. 


\section{Class of non-linear problems}

Let $H_{u}$ and $H_{\phi}$ be real Hilbert spaces of vectors $u$ and scalars $\phi$ defined on some compact connected subset $V$ of Euclidean space $E^{n}$ with smooth boundary $\partial V=\partial V_{1}+\partial V_{2}$. The inner products on $H_{u}$ and $H_{\phi}$ are denoted by [,] and $\langle$.$\rangle respectively. A linear operator T$ maps $H_{\phi}$ into $H_{u}$. The domain of $T$ is dense in $H_{\phi}$, and we assume that an operator $T^{*}$ is defined on $H_{u}$, mapping $H_{u}$ into $H_{\phi}$, where $T^{*}$ is the formal adjoint of $T$ such that

$$
[u, T \phi]=\left\langle T^{*} u, \phi\right\rangle+[u, \sigma \phi]_{\partial v} .
$$

Here $\sigma$ is an operator mapping $H_{\phi}$ into $H_{u}$ on $\partial V$, with adjoint $\sigma^{*}$ defined by

$$
[u, \sigma \phi]_{\partial V}=\left\langle\sigma^{*} u, \phi\right\rangle_{\partial V} .
$$

A standard example of these inner products and operators is given by

$$
\left.\begin{array}{rl}
{[u, v]} & =\int_{V} \mathbf{u} \cdot \mathbf{v} d V, \quad[u, v]_{\partial V}=\int_{\partial V} \mathbf{u} \cdot \mathbf{v} d B, \\
\langle\phi, \psi\rangle & =\int_{V} \phi \psi d V, \quad\langle\phi, \psi\rangle_{\partial V}=\int_{\partial V} \phi \psi d B, \\
T & =\text { grad, } \quad T^{*}=-\operatorname{div}, \quad \sigma=\mathbf{n}, \quad \sigma^{*}=\mathbf{n} .,
\end{array}\right\}
$$

which we shall use later. More general examples arise when the inner products involve weighting factors or when the operators are more complicated, but we do not need the details here.

We shall consider boundary value problems described by equations of the form

$$
\begin{aligned}
T^{*}[h(T \phi)]+a \phi & =b \quad \text { in } V, \\
\phi & =\phi_{B} \text { on } \partial V_{1}, \\
\sigma^{*} h(T \phi) & =\sigma^{*} u_{B} \text { on } \partial V_{2},
\end{aligned}
$$

where $h$ is a known function of vectors $u$ in $H_{u}$ with an inverse, $a>0$ and $b$ are given functions of position in $V$, and $\phi_{B}$ and $u_{B}$ are prescribed functions on parts $\partial V_{1}$ and $\partial V_{2}$ of the boundary. The boundary conditions here correspond to Dirichlet and Neumann types on $\partial V_{1}$ and $\partial V_{2}$ respectively. We assume the existence of a scalar solution $\phi$ of (2.4)-(2.6). Conditions for uniqueness are discussed in the next section.

\section{Geometrical formulation}

To derive hypercircle results for the class of problems in (2.4)-(2.6) we first employ the basic procedure of splitting equation (2.4) into a pair of canonical equations by taking

$$
\begin{gathered}
T \phi=k(u), \quad \phi=\phi_{B} \quad \text { on } \partial V_{1}, \\
T^{*} u=b-a \phi \quad \text { in } V, \quad \sigma^{*} u=\sigma^{*} u_{B} \text { on } \partial V_{2},
\end{gathered}
$$


where $k$ is the inverse of $h$. A solution ( $u, \phi)$ of these equations satisfies (2.4)-(2.6).

Next we introduce a new function space ( $F$-space like phase space) with points $s_{i}=\left(u_{i}, \phi_{i}\right)$, but without metric for the moment. Let $s=(u, \phi)$ denote the solution of (3.1) and (3.2). Then $s$ is the intersection of two manifolds:

(1) a non-linear manifold $\Omega_{1}$ defined by

$$
T \phi_{1}=k\left(u_{1}\right), \quad \phi_{1}=\phi_{B} \quad \text { on } \partial V_{1}
$$

(2) a linear affine subspace $\Omega_{2}$ defined by

$$
T^{*} u_{2}=b-a \phi_{2} \quad \text { in } V, \quad \sigma^{*} u_{2}=\sigma^{*} u_{B} \quad \text { on } \partial V_{2} .
$$

Now we shall define the scalar or inner product for any two vectors $s_{i}$ and $s_{j}$ in $F$-space by

$$
s_{i} . s_{j}=\left[u_{i}, \beta u_{j}\right]+\left\langle\phi_{i}, a \phi_{j}\right\rangle,
$$

where $\beta$ is some positive constant. Since $a$ is strictly positive, the metric is positive definite if $\beta>0$. If $\beta=0$ we work instead with the function space $\left\{\phi_{i}\right\}$.

If $s=(u, \phi)$ is the solution, $s_{1}=\left(u_{1}, \phi_{1}\right)$ a point in $\Omega_{1}$ and $s_{2}=\left(u_{2}, \phi_{2}\right)$ a point in $\Omega_{2}$, we have

$$
\begin{aligned}
\left(s-s_{1}\right) \cdot\left(s-s_{2}\right) & =\left[u-u_{1}, \beta\left(u-u_{2}\right)\right]+\left\langle\phi-\phi_{1}, a\left(\phi-\phi_{2}\right)\right\rangle \\
& =\left[u-u_{1}, \beta\left(u-u_{2}\right)\right]-\left\langle\phi-\phi_{1}, T^{*}\left(u-u_{2}\right)\right\rangle \\
& =\left[u-u_{2}, \beta\left(u-u_{1}\right)-T\left(\phi-\phi_{1}\right)\right],
\end{aligned}
$$

by (2.1) and using the fact that the boundary terms vanish since $\phi_{1}=\phi=\phi_{B}$ on $\partial V_{1}$ and $\sigma^{*} u_{2}=\sigma^{*} u=\sigma^{*} u_{B}$ on $\partial V_{2}$. By (3.3) we therefore have

$$
\left(s-s_{1}\right) \cdot\left(s-s_{2}\right)=-\left[u-u_{2}, k(u)-k\left(u_{1}\right)-\beta\left(u-u_{1}\right)\right] .
$$

To obtain a hypercircle result from this we must ensure that the right-hand side is non-positive. Such a result depends upon our choice of $\beta$ and the properties of $k(u)$. If $k(u)$ is monotone, so that

$$
[u-v, k(u)-k(v)] \geqslant 0
$$

for functions $u$ and $v$ in $H_{u}$, then by requiring

and choosing

$$
u_{2}=u_{1} \text {, }
$$

we obtain

$$
\beta=0 \text {, }
$$

$$
\left(s-s_{1}\right) \cdot\left(s-s_{2}\right) \leqslant 0 .
$$

Hence the solution $s$ lies inside (or on) a hypersphere

$$
(x-c)^{2}=R^{2} \text {, }
$$


with centre $c$ and radius $R$ given by

where $s_{i}^{2}=s_{i} \cdot s_{i}$.

$$
2 c=s_{1}+s_{2}, \quad 4 R^{2}=\left(s_{1}-s_{1}\right)^{2},
$$

Let us now examine the case when $k(u)$ satisfies stronger conditions. Thus suppose that $k(u)$ is strongly monotone, so that

$$
[u-v, k(u)-k(v)] \geqslant\|u-v\| \bar{\beta}(\|u-v\|),
$$

where $\|u\|=[u, u]^{\frac{1}{2}}$ and $\bar{\beta}(t)$ is an increasing function of the real variable $t \geqslant 0$ such that $\bar{\beta}(0)=0$ and $\bar{\beta}(t) \rightarrow \infty$ as $t \rightarrow \infty$. Then if we again require

it follows from (3.6) that

$$
u_{2}=u_{1} \text {, }
$$

$$
\left(s-s_{1}\right) \cdot\left(s-s_{2}\right) \leqslant \beta\left\|u-u_{1}\right\|^{2}-\left\|u-u_{1}\right\| \bar{\beta}\left(\left\|u-u_{1}\right\|\right) .
$$

Choosing the constant $\beta$ in (3.5) so that

we obtain

$$
0 \leqslant \beta \leqslant t^{-1} \bar{\beta}(t), \quad t \geqslant 0,
$$

$$
\left(s-s_{1}\right) \cdot\left(s-s_{2}\right) \leqslant 0,
$$

as in (3.10), and hence $s$ lies inside (or on) the hypersphere (3.11). Because of condition (3.14), the radius $R$ of the hypersphere, given by

$$
4 R^{2}=\left(s_{1}-s_{2}\right)^{2}=\left[u_{1}-u_{2}, \beta\left(u_{1}-u_{2}\right)\right]+\left\langle\phi_{1}-\phi_{2}, a\left(\phi_{1}-\phi_{2}\right)\right\rangle,
$$

is independent of the choice of $\beta$ allowed by (3.16). Other parameters however, such as $c^{2}=R^{2}+s_{1} . s_{2}$, do depend on the value of $\beta$ used in the scalar product (3.5).

If $k(u)$ possesses an abstract derivative $k^{\prime}(u)$, the monotone nature of $k(u)$ may be established by using the mean value theorem

$$
\left[u-u_{1}, k(u)-k\left(u_{1}\right)\right]=\left[u-u_{1}, k^{\prime}(\bar{u})\left(u-u_{1}\right)\right],
$$

where $\bar{u}=u_{1}+\varepsilon\left(u-u_{1}\right), 0<\varepsilon<1$. In some cases $k^{\prime}$ is bounded below so that

$$
\left[u_{i}, k^{\prime}\left(u_{j}\right) u_{i}\right] \geqslant \beta_{1}\left[u_{i}, u_{i}\right]>0
$$

for all non-zero $u_{i}, u_{j}$ in $H_{u}$. It then follows that $k(u)$ is strongly monotone with

$$
\bar{\beta}(t)=\beta_{1} t .
$$

In this case equation (3.16) becomes

$$
0 \leqslant \beta \leqslant \beta_{1} \text {. }
$$

Linear problems correspond to

$$
k(u)=u
$$

which is strongly monotone with $\beta_{1}=1$. If we now choose $\beta=1$, equation (3.6) shows directly that the solution $s$ lies on the hypersphere (3.11) and condition (3.8) 
or (3.14) need not be imposed. Thus we recover the original hypercircle results of Synge [6] for linear boundary value problems with Dirichlet or Neumann boundary conditions.

For our class of problems in (3.1) and (3.2) we can now establish uniqueness of solution $s$ under the weakest assumptions made here, namely that $a$ is positive and $k(u)$ is monotone. To see this we note that if $s_{1}=s_{2}=$ one solution, then any other solution is contained in a hypersphere of radius $R=0$ about $c=s_{1}$ and hence is again $s_{1}$.

\section{An example}

To illustrate these results we take the non-linear partial differential equation problem described by the equations

with

$$
\operatorname{div}\{D(\phi) \operatorname{grad} \phi\}=\phi \quad \text { in } V,
$$

where

$$
\text { n. } D(\phi) \operatorname{grad} \phi=\cos \gamma \quad \text { on } \partial V \text {, }
$$

$$
D(\phi)=\left(1+|\operatorname{grad} \phi|^{2}\right)^{-\frac{1}{2}} \text {. }
$$

This problem arises in the determination of an equilibrium-free surface $S$ of a liquid that partially fills a cylindrical container under surface forces, gravitational forces and boundary adhesion (see [1]). Here $\phi=\phi(x, y)$ represents the height of the capillary surface, $\gamma$ is the angle of intersection of $S$ and the cylindrical container (measured interior to the liquid) and $\mathbf{n}$ is the outward unit normal field on the boundary $\partial V$ of the cross-section $V$ of the cylinder. For boundaries $\partial V$ of class $C^{4}$ and $\gamma>0$, it has been shown [5] that a solution of the problem exists and is unique. We shall suppose that these conditions are satisfied.

To obtain hypercircle estimates for this problem we set

$$
D(\phi) \operatorname{grad} \phi=\mathbf{u} \quad(\text { note } \mathbf{u} . \mathbf{u} \leqslant 1),
$$

and then (4.1) and (4.2) take the canonical form

$$
\begin{aligned}
& \operatorname{grad} \phi=\mathbf{u}(1-\mathbf{u} \cdot \mathbf{u})^{-\frac{1}{2}}, \\
& -\operatorname{div} \mathbf{u}=\phi \quad \text { in } V, \quad \mathbf{n} \cdot \mathbf{u}=\cos \gamma \quad \text { on } \partial V .
\end{aligned}
$$

Comparing with (3.1) and (3.2) we see that

$$
\begin{aligned}
T & =\operatorname{grad}, \quad T^{*}=-\operatorname{div}, \quad \sigma^{*}=\mathbf{n} ., \\
\partial V_{1} & =0, \quad \partial V_{2}=\partial V \\
k(\mathbf{u}) & =\mathbf{u}(1-\mathbf{u} \cdot \mathbf{u})^{-\frac{1}{2}} \\
a & =1, \quad b=0 .
\end{aligned}
$$


Now we examine the function $k(\mathbf{u})$ in (4.9) for monotone properties. Consider

$$
\begin{aligned}
K & =[\mathbf{u}-\mathbf{v}, k(\mathbf{u})-k(\mathbf{v})] \\
& =\left[\mathbf{w}, k^{\prime}(\overline{\mathbf{u}}) \mathbf{w}\right],
\end{aligned}
$$

where $\mathbf{w}=\mathbf{u}-\mathbf{v}$ and $\overline{\mathbf{u}}=\mathbf{v}+\varepsilon(\mathbf{u}-\mathbf{v}), 0<\varepsilon<1$. Since

we have

$$
k^{\prime}(\mathbf{u})=\frac{1}{(1-\mathbf{u} \cdot \mathbf{u})^{\frac{1}{2}}}+\frac{\mathbf{u u}}{(1-\mathbf{u} \cdot \mathbf{u})^{3 / 2}},
$$

$$
\begin{aligned}
K & =\left[\mathbf{w},(1-\overline{\mathbf{u}} \cdot \overline{\mathbf{u}})^{-\frac{1}{2}} \mathbf{w}\right]+\left[\mathbf{w},(1-\overline{\mathbf{u}} \cdot \overline{\mathbf{u}})^{-3 / 2} \overline{\mathbf{u}} \overline{\mathbf{u}} \cdot \mathbf{w}\right] \\
\geqslant & {\left[\mathbf{w},(1-\overline{\mathbf{u}} \cdot \overline{\mathbf{u}})^{-\frac{1}{2}} \mathbf{w}\right] } \\
\geqslant & {[\mathbf{w}, \mathbf{w}] } \\
& =\|\mathbf{u}-\mathbf{v}\|^{2} .
\end{aligned}
$$

This shows that $k(\mathbf{u})$ is strongly monotone, a suitable function $\bar{\beta}(t)$ in (3.13) being

$$
\bar{\beta}(t)=t
$$

By (3.20) and (3.21) we see that possible values of $\beta$ are given by $0 \leqslant \beta \leqslant 1$. For definiteness let us choose

$$
\beta=1,
$$

and the scalar product (3.5) for this problem is then

$$
s_{i} . s_{j}=\left[\mathbf{u}_{i}, \mathbf{u}_{j}\right]+\left\langle\phi_{t}, \phi_{j}\right\rangle .
$$

Since for this problem $a$ is positive and $k(\mathrm{u})$ is strongly monotone, it follows from Section 3 that the solution $s$ is unique.

We shall consider the case of a liquid in a cylindrical container with circular cross-section of radius one. We take very simple trial functions $s_{1}$ in $\Omega_{1}$ and $s_{2}$ in $\Omega_{2}$ with $s_{1}=\left(\mathbf{u}_{1}, \phi_{1}\right)$ and $s_{2}=\left(\mathbf{u}_{2}, \phi_{2}\right)$ where

and

$$
\begin{aligned}
& \phi_{1}=\alpha-\left(\sec ^{2} \gamma-r^{2}\right)^{\frac{1}{2}}, \\
& \mathbf{u}_{1}=\mathbf{n} r \cos \gamma,
\end{aligned}
$$

$$
\begin{aligned}
& \phi_{2}=2 \cos \gamma, \\
& \mathbf{u}_{2}=\mathbf{n} r \cos \gamma .
\end{aligned}
$$

Here $u_{2}=u_{1}$, as required by (3.14), and $\alpha$ is a free parameter which is determined by minimizing the radius $R$ of the hypersphere given in this case by

$$
4 R^{2}=\left(s_{1}-s_{2}\right)^{2}=\int_{0}^{1} \int_{0}^{2 \pi}\left(\phi_{1}-\phi_{2}\right)^{2} r d r d \theta
$$

in plane polar coordinates. With these trial functions the optimization can be 
carried out analytically. Taking the contact angle $\gamma$ equal to $\pi / 3$, we obtain the results

$$
\alpha=2.869, \quad R=0.074, \quad|c|=1.88 \text {. }
$$

The size of $R$ here indicates that our simple optimized solution (4.17) is reasonably good. From it we estimate, for example, that the height of the free surface $S$ at $r=0$ is approximately 0.869 , which is in quite good agreement with the value 0.887 given by a more elaborate variational calculation [2].

\section{Concluding remarks}

For problems of the form

$$
T^{*}[h(T \phi)]=g(\phi),
$$

hypercircle estimates are now available in the three cases

(i) $h$ and $g$ linear [6],

(ii) $h$ linear, $g$ non-linear [4],

(iii) $h$ non-linear, $g$ linear (this paper).

Cases (ii) and (iii) of (5.1) are basically different, but they reflect the formal symmetry that exists in the pair of associated canonical equations. For the case (iv) $h$ non-linear, $g$ non-linear,

which provided the original motivation for this investigation, it does not appear possible to establish similar hypercircle results. A formulation of case (iv) based on the distance geometry of [3] is possible, however, and this involves a distance function $d_{i j}$ given by

$$
d_{i j}^{2}=\left[u_{i}-u_{j}, k\left(u_{i}\right)-k\left(u_{j}\right)\right]-\left\langle\phi_{i}-\phi_{j}, g\left(\phi_{i}\right)-g\left(\phi_{j}\right)\right\rangle,
$$

where $k=h^{-1}$ and $-g$ are strictly monotone. This geometry enables us to calculate the distance between points in the non-linear manifolds $\Omega_{1}$ and $\Omega_{2}$, but it lacks the metric space structure of the hypercircle geometry.

\section{References}

[1] N. K. Adam, The physics and chemistry of surfaces (New York: Dover, 1968).

[2] N. Anderson and A. M. Arthurs, "Maximum and minimum principles for capillary surface problems with prescribed contact angle", J. Austral. Math. Soc. B 20 (1978), 285-289.

[3] A. M. Arthurs, J. Math. Anal. Appl. 60 (1977), 483-492.

[4] A. M. Arthurs and V. G. Hart, "The method of the hypercircle for a class of nonlinear equations", J. Austral. Math. Soc. B 21 (1979), 75-83.

[5] J. Spruch, Comm. Pure Appl. Math. 28 (1975), 189-200.

[6] J. L. Synge, The hypercircle in mathematical physics (Cambridge University Press, 1957). 
Department of Mathematics

University of York

York, Y01 5DD

England 